Cell Research (1999), 9, 189-199

\title{
Expression of a soluble form of CTLA4 on macrophage and its biological activity
}

GAO Yue Hua1, Pichao WANG1, Kuniaki TAKAGI2, Osamu ShimoZato3, Hideo Yagita3, Tohru

OKIGAKI4, Masatoshi MATASUMURA1,*

1 Institute of Applied Biochemistry, University of Tsukuba, Ibaraki 305-8572, Japan

2 Department of Environmental Biochemistry, School of Pharmaceutical Science, Univer sity of Shizuoka, Shizuoka 422, Japan

3 Department of Immunology, Juntendo University School of Medicine, Tokyo 113, Japan

4 Division of Molecular and Cell Biology, Shigei Medical Research Institute, Okayama 701-0202, Japan

\section{ABSTRACT}

Interaction between cytotoxic $\mathrm{T}$ lymphocyte-asso- ciated antigen-4 (CTLA4, CD152) and B7 molecules (B7-1 and B7-2) is of importance in the cellular events of lymphocyte, including antigen-specific T-cell activation and induction of autoreactive T-cell. We describe here the first introduction of a murine soluble CTLA4 gene, CTLA4Ig, to Mm1 cells, a macrophagic cell line. CTLA4Ig was successfully expressed on Mm1 cells and the expressed CTLA4Ig was found to be functionally active in their binding to B7 molecules by flow cytometry and immunofl- uorescence studies. The biological activity of CTLA4Ig from the transfected Mm1 cells was studied and showed inhibitory activity on mixed lymphocyte culture. A high CTLA4Ig producing macrophagic cell line was obtained. As $\mathrm{Mm} 1$ cells were regarded as difficult for gene transfection and there had so far been no report on expression of CTLA4Ig gene on Mm1 cells, these results suggested that the CTLA4Ig ex-

* Corresponding author: Masatoshi Matsumura, Institute of Applied Biochemistry University of Tsukuba, 1-1-1 Tennodai, Tsukuba Ibaraki 305-8572, Japan Phone: +81 298(53)6581 Fax: +81 298(53)4605 e-mail:aquacel@sakura.cc.tsukuba.ac.jp 
Expression of CTLA4 on Mm1 and its biological activity

pressing Mm1 cells could be useful for analysis of CTLA4 and B7 molecule interaction in both macrophage and T-cell.

Key words: Macrophage, fusion protein, CTLA4, B7, transfection.

\section{INTRODUCTION}

It is recently reported that $\mathrm{B} 7$ and CD28/CTLA4 pathway plays a critical role in the activation of T-cell[1-3], as well as in the onset of autoimmunity both in human and murine model of autoimmunity[4-7]. B7 molecules are expressed on a variety of cell types, including dentritic cells, B cells, T-cells and macrophages[8-11]. CTLA4Ig, a soluble form of CTLA4, could block B7 and CD28/CTLA4 pathway and results in the inhibitation of Tcell activation and autoimmune response[12-19].

The macrophagic Mm1 cell line was regarded as a good model for studying macrophage differentiation and cellular functional expression[20-23]. Mm1 cells were derived from M1 cells that were established from a spontaneous myeloid leukemia of SL strain mice [24], [25]. Previous studies revealed that Mm1 cells possessed some, but not all, mature macrophage phenotypes and immunological functions, for example, they have Fc receptor and phagocytic capacity, but lack accessory cell activity and Ia antigen[26],[27]. The involvement of tumor necrosis factor and colony stimulating factor in the differentiation of macrophage were also investigated recently[23],[28],[29].

Like other macrophage lines or primary macrophages[30], Mm1 cells were regarded to be difficult for transfection and few attempts have been made for gene transfection in Mm1 cells. To study whether Mm1 cells can be successfully transfected by CTLA4Ig gene, we made a trial to transfect a murine CTLA4Ig gene into Mm1 cells. The expression of CTLA4Ig from transfected Mm1 cells was characterized in this study. We thus showed that CTLA4Ig gene was successfully transfected into Mm1 cells and expressed positively and stably. Moreover, we confirmed the biological activities of CTLA4Ig produced by the transfected $\mathrm{Mm} 1$ cells. Our study resulted in the establishment of a new macrophage line and indicated possible approach to further genetic engineering studies of $\mathrm{Mm} 1$ cells.

\section{MATERIALS AND METHODS}

\section{Cell line, cell culture and transfection}

Mm1 cell line was purchased from Riken Cell Bank (Tsukuba, Japan). B7-1 and B7-2 transfectants were previously described[31], [32]. All the cell cultures, unless otherwise stated, were carried out with complete RPMI 1640 medium, i.e., RPMI 1640 (Nissui Corp., Tokyo, Japan) supplemented with L-glutamine, $10 \%$ heat-inactivated fetal calf serum (FCS, Sanko Junyaku Co., Ltd., Tokyo, Japan), $100 \mathrm{U} / \mathrm{ml}$ penicillin and $0.1 \mathrm{mg} / \mathrm{ml}$ streptomycin.

Plasmid PM/CTLA4Ig containing CTLA4Ig cDNA from mouse library of CTLA4 and 
IgG2aFc, and pMKITneo vector that was kindly provided by K. Maruyama (Tokyo Medical and Dental University). Stable expression of CTLA4Ig DNA in Mm1 cells was performed by using Gene Pulser (Bio-Rad Laboratories, Hercules, CA) according to the method of Wang et al.[33] with slight modifications. Briefly, the constructed CTLA4Ig plasmid was purified by centrifugation in cesium chloride and was linearized with restriction enzyme Sma I (Wako Pure Chemical Industries, Japan) digestion. $10 \mu \mathrm{g}$ DNA in $10 \mu \mathrm{C} \mathrm{Ca}^{2+}$ and $\mathrm{Mg}^{2+}$ free phosphate-buffered saline (PBS) was mixed with $3 \times 10^{6} \mathrm{Mm} 1$ cells suspended in $0.2 \mathrm{ml}$ culture medium. The DNA and the cells were allowed to sit for $10 \mathrm{~min}$ at room temperature in a chamber (Bio-Rad) and then subjected to an electric pulse at $300 \mathrm{~V}$ and $960 \mu \mathrm{F}$. Cells were allowed to sit for 10 min further at $0{ }^{\circ} \mathrm{C}$ before adding $10 \mathrm{ml}$ culture medium. The cells were then seeded in 96 -well-microplate (Nunc, Roskilde, Denmark) with a cell density of $3 \times 10^{4}$ cells per well in culture medium containing 0 . $5 \mathrm{mg} / \mathrm{ml}$ neomycin G418 (Boehringer Mannheim Biochemica, Germany) for selection of positive clones. Cells were grown for 10 days until colonies appeared without change of culture medium. Culture supernatant from the microplate was screened by an enzyme-linked immunosorbent assay (ELISA, described later) for CTLA4Ig, then the positive colonies were subsequently cloned twice by limiting dilution to obtain high CTLA4Ig producing clones.

\section{Sandwich ELISA, SDS-polyacrylamide gel electrophoresis (SDS- PAGE) and Western blotting}

Sandwich ELISA for CTLA4Ig was performed as follows. Samples $(0.1 \mathrm{ml})$ were plated to wells of an ELISA plate (Nunc, MaxiSorp) coated with hamster anti-mouse CTLA4 monoclonal antibody (mAb, clone 4F-10, PharMingen, San Diego, CA), and incubated at $37^{\circ} \mathrm{C}$ for $1 \mathrm{~h}$, and the plate was washed three times with PBS containing 0.5\% Tween-20 (T-PBS). Then, $0.1 \mathrm{ml}$ of horseradish peroxidase(HRP)-labeled polyclonal rabbit anti-mouse IgG2a ( $\gamma 2$ a chain) antibody (1:500 dilution, Organon Teknika Corp., Durham, NC) was added and incubated at $37{ }^{\circ} \mathrm{C}$ for $1 \mathrm{~h}$. After washing, substrate solution containing O-phenylenediamine was added and colourimetric reaction was terminated by $1 \mathrm{~N}$ phosphoric acid. Spectraphotometric measurement was carried out with a microplate reader (Bio-Rad) at $490 \mathrm{~nm}$. The CTLA4Ig amounts were calibrated with a standard curve of CTLA4Ig protein that was purified from culture supernatant of a stably murine CTLA4Ig transfected Chinese hamster ovary (CHO) cell line established in our preliminary studies (data not reported, CHO was from Riken Cell Bank) with an affinity column of protein A-Sepharose (Pharmacia Sweden).

SDS-PAGE was done according to the method of Laemmli[34]. Mouse IgG1 and IgG2a were used as control proteins. Samples were run under both reducing (+2-Mercaptoethanol, 2ME) and non-reducing (-2ME) conditions. After the SDS-PAGE, the proteins were stained by Coomassie Brilliant Blue, or blotted onto nitrocellulose membranes (pore size, $0.2 \mu \mathrm{m}$ ). The membranes were soaked in 5\% skim milk (in PBS) and then treated either with HRP-conjugated goat anti-mouse Ig or rabbit anti-mouse IgG2a ( $\gamma 2$ a chain) antibody alone, or with 1:1000 dilution of the primary antibody (anti-mouse CTLA4 mAb, clone 4F-10) and 1:2000 dilution of the second antibody (HRP-conjugated goat anti-hamster IgG, Southern Biotechnology Associates, Inc., Birmingham, AL). After washing, the membranes were treated with the substrate (3,3', 5,5' tetramethylbenzidine, TMB, Kirkegaar and Perry Laboratories Inc., Gaithersburg, MD) until bluish purple color developed.

\section{Flow cytometry and immunofluorescence}

$1 \times 10^{6}$ B7-1 or B7-2 transfectants were incubated in culture media from CTLA4Ig transfected $\mathrm{Mm} 1$ cells or in PBS served as control for $30 \mathrm{~min}$ at $4{ }^{\circ} \mathrm{C}$. After washing, the cells were incubated with fluorescein isothiocyanate(FITC)-conjugated goat anti-mouse Ig's antibody (1:200 dilution, Bio Source Int., Camarillo, CA). The cells incubated with FITC-conjugated rat anti-mouse CD80 or CD86 mAb alone were conducted for the confirmation of B7-1 and B7-2 antigen. After washing, 
Expression of CTLA4 on Mm1 and its biological activity

the cells were analyzed on FACScan (Becton Dickinson, Lincoln Park, NJ). These cells were also observed by an Olympus LSM-GB200 microscope (Olympus, Tokyo, Japan) with phase-contrast, conventional fluorescence and confocal laser scanning modes.

\section{Mixed lymphocyte culture $(M L C)$}

Spleen cells were harvested and suspended in culture medium. $2.5 \times 10^{5} \mathrm{BALB} / \mathrm{c}\left(\mathrm{H}-2^{d}\right)$ responder cells were cocultured with $2.5 \times 10^{5}$ mitomycin C-treated C57BL/6 (H-2 $\left.{ }^{b}\right)$ stimulator cells. The cells were incubated in the presence or absence, served as control experiments, of the culture supernatants from CTLA4Ig transfected Mm1 cells. Incubations were done in quadruplicate in a 96 -well microplate for $5 \mathrm{~d}$ at $37^{\circ} \mathrm{C}$. The cultures were pulsed with $1 \mathrm{~g}$ Ci of $\left[{ }^{3} \mathrm{H}\right]$-thymidine (ICN Biomedicals Inc., Costa Mesa, CA) 18 hours before the termination of the culture and aliquots were harvested on glass fiber filters. Determination of $\left[{ }^{3} \mathrm{H}\right]$-thymidine incorporation was measured using a liquid scintillation counter (Beckman, Palo Alto, CA). Results were expressed as mean counts per minute $(\mathrm{cpm}) \pm$ standard division (S.D.). The data were analyzed statistically by Student's t-test.

\section{RESULTS AND DISCUSSION}

Successful expression of CTLA4Ig gene on Mm1 cells

CTLA4Ig is a soluble fusion protein consisting of extracellular domain of CTLA4 and IgG Fc region. The plasmid PM/CTLA4Ig used in this study encodes the extracellular portion of mouse CTLA4 and mouse IgG2aFc. In the initial attempts to transfect the plasmid into the $\mathrm{Mm} 1$ cells by using electroporation, variations on setting the power supplies and capacitance as well as the cell number and DNA concentration have been done. Alternatively, the temperature was also changed. We found that the optimal condition for $\mathrm{Mm} 1$ cell transfection was $300 \mathrm{~V}$ and $960 \mu \mathrm{F}$ (the time constant was $30 \mathrm{msec}$ ) performed at room temperature.

After the electroporation, colonies that showed resistance to G418 were screened by sandwich ELISA for mouse CTLA4Ig as described in materials and methods. A high CTLA4Ig producing clone, E522.7.50, was obtained. To confirm molecular weight of the protein released from E522.7.50, the culture supernatant was passed over a protein ASepharose column and an eluate was subjected to electrophoresis. A single protein band consisted with molecular weight of 110,000 daltons without reduction was observed (Fig 1, lanes 3, 6, 9 and 12). Western blotting also showed a single band at a molecular weight of 55,000 daltons with reduction (Fig 1, lane 15), indicating that the purified protein was a formation of homodimer. Moreover, Western blotting analysis showed that anti-mouse IgG2a ( $\gamma 2 \mathrm{a}$ chain) antibody and anti-mouse CTLA4 mAb bound to the purified proteins specifically (Fig 1, lanes 9,12 and 15). These results confirmed the isotype specificity of the fusion protein of CTLA4Ig, i.e., mouse CTLA4 and IgG2aFc, released from the CTLA4Ig transfected Mm1 cells, which was agreed with the findings by Lane et. al [12].

CTLA4Ig from transfected Mm1 cells binds effectively to B7 molecules

It is reported that both B7-1 and B7-2 are the ligands for CTLA4 and expressed on cell surface as membrane antigens[8-11]. CTLA4Ig binds these molecules with very high affinity[1]. To examine whether CTLA4Ig from E522.7.50 could bind the B-7 molecules, 


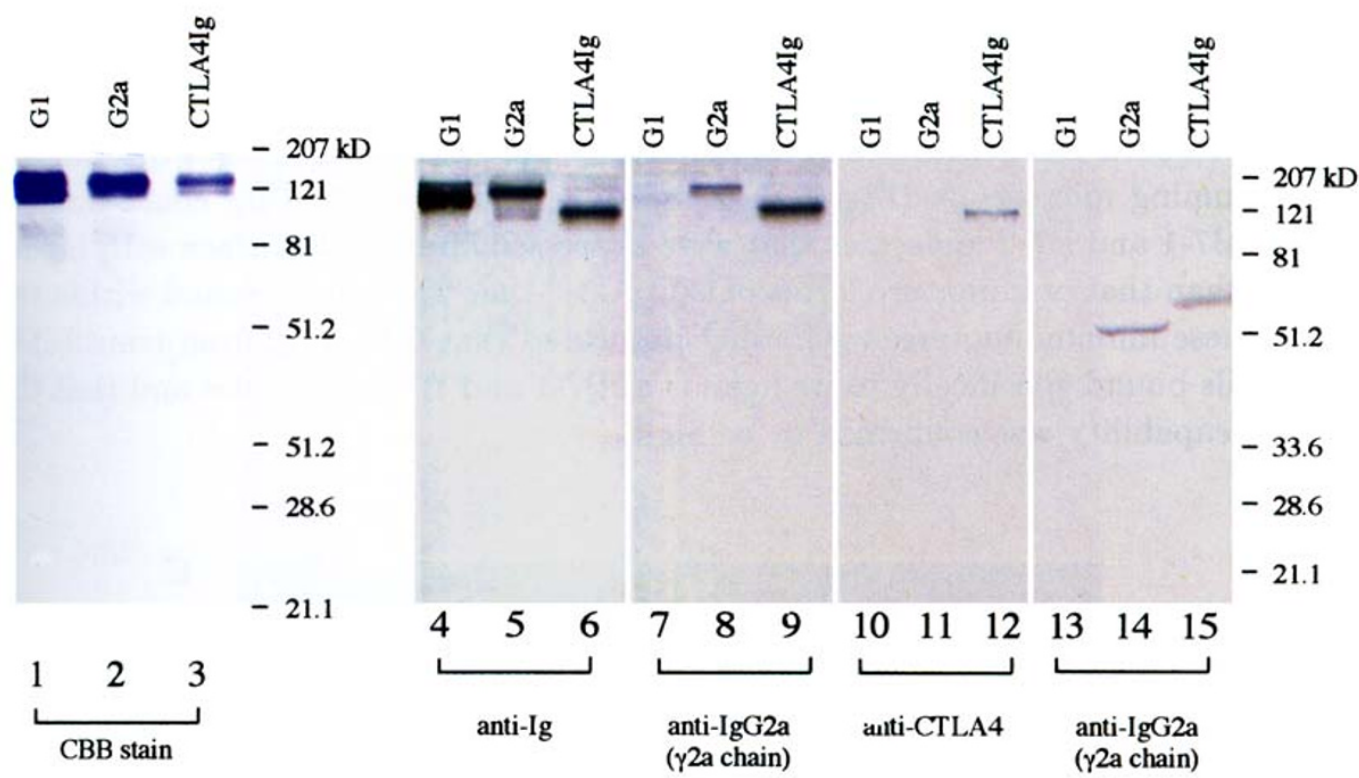

Fig 1.

SDS-PAGE and Western blotting analysis of CTLA4Ig protein from Mm1 cells transfected with CTLA4Ig DNA.

Plasmid encoding a fusion protein of CTLA4Ig was transfected into Mm1 cells. After screening with ELISA, a high CTLA4Ig producing clone, E522.7.50, was selected. Culture media was collected and IgCg-containing proteins were purified by protein A affinity chromatography as described in materials and methods. Aliquots $(2 \mu \mathrm{g})$ of protein A adherent fraction, mouse IgG1 and IgG2a were subjected to SDS-PAGE (12\% polyacrylamide) with or without prior reduction with $2 \mathrm{ME}$. The proteins were stained by Coomassie Brilliant Blue (lanes 1-3) or transferred to a nitrocellulose membrane and stained with anti-Ig, anti-IgG2a ( $\gamma 2 \mathrm{a}$ chain) and anti-CTLA4 (lanes 4-15) antibodies, respectively. The positions of molecular weight markers are shown on the right of both panels. Note that CTLA4Ig from transfected Mm1 cells was specifically recognized by anti-Ig $\gamma 2 \mathrm{a}$ and anti-CTLA4 antibodies.

we performed indirect immunofluorescence staining of B7-1 and B7-2 transfectants with CTLA4Ig. Culture media from E522.7.50 cells was used as a source of CTLA4Ig, while in later experiments the purified protein was used. No difference was found between these two sources of CTLA4Ig (data not shown). Binding was detected by addition of culture media to B7-1 and B7-2 transfected cells and by subsequent addition of a second step reagent of FITC-conjugated goat anti-mouse Ig. Fig 2 showed the results of flow cytometry. B7-1 and B7-2 transfectants express a high level of mouse B7-1 and B7-2 on the cell surface respectively (Fig $2 \mathrm{~A}$ and $\mathrm{C}$ ). An increasing fluorescence intensity was found in culture media-treated groups, compared with that treated with PBS (Fig 2 B and D). The cells were studied further by phase-contrast (Fig 3 a-d) and conventional immunofluorescence microscopy (Fig 3 A-D). Simultaneously, results showed positively stained struc- 
Expression of CTLA4 on Mm1 and its biological activity

tures on the surface of B7-1 and B7-2 cells in culture media-treated group (Fig 3 B and D), whereas the cells treated with PBS served as control were not stained or very weakly stained if any (Fig $3 \mathrm{~A}$ and $\mathrm{C}$ ). The staining was most prominent in the cell surface region and it could be seen more clearly by epifluorescence optical section images by confocal laser scanning microscopy (Fig 4 A), suggesting that the CTLA4Ig could bind to mature B7-1 and B7-2 molecules that were expressed on the cell surface with higher affinity than that of immature forms of CTLA4Ig, that was usually found within the cells. These immunofluorescence findings indicated that CTLA4Ig from transfected Mm1 cells bound specifically to its ligands of B7-1 and B7-2 molecules and that the binding capability was confirmed to be high.

Fig 2.

Binding of CTLA4Ig to B7-1 and B7-2 molecules.

Expression of B7-1 and B7-2 antigen was confirmed at first by either anti-CD80 or anti-CD86 mAb (A and C). B7-1 and B7-2 cells were incubated for 30 min with the culture medium of clone E522.7.50 and detected with the second antibody of goat anti-mouse Ig-FITC (B and D). Background fluorescence of the cells incubated in PBS followed by the second antibody is shown by the black-lacquered areas in all experiments. A total of 10 , 000 stained cells was analyzed. A and B: B7-1 transfectant; C and D: B7-2 transfectant.

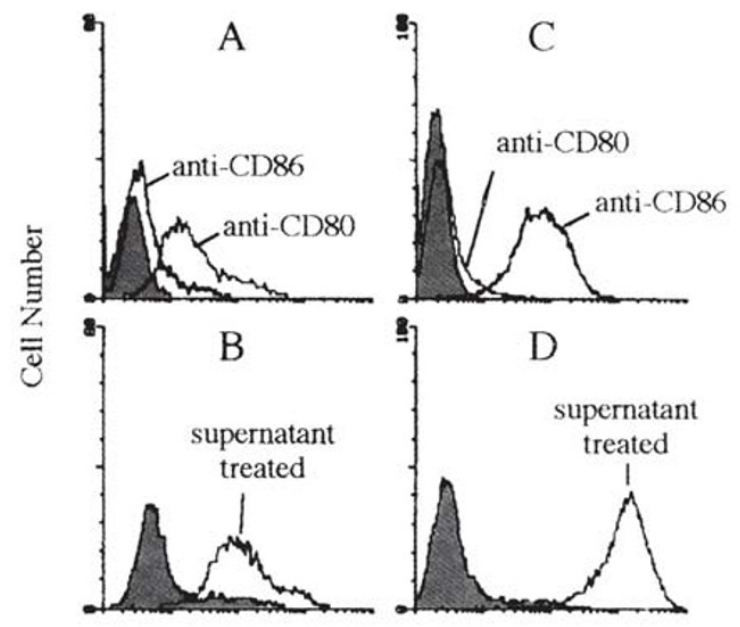

$\log 10$ fluorescence

\section{CTLA4Ig from transfected Mm1 cells inhibits T-cell activation in mixed lymphocyte culture}

In order to examine the biological activity of CTLA4Ig from transfected Mm1 cells, we then verified the biological ability of CTLA4Ig in terms of inhibitory effect on T-cell

\section{$\triangleright$ Fig 3.}

Immunofluorescence microscopic observation of the cells stained with CTLA4Ig protein.

B7-1 (a, b, A, B) and B7-2 (c, d, C, D) cells were incubated in culture medium of clone E522. 7. 50 (b, B, d, D) or PBS (a, A, d, D) served as control and subsequently incubated with a FITC-labeled second antibody. Observation was conducted by an Olympus LSM-GB200 microscope with phase-contrast (a-d) and conventional fluorescence (A-D) modes. Note that positively stained structures on the cell membrane in culture medium-treated group (Fig 3 B and D), whereas the controls were not stained or very weakly stained if any (Fig $3 \mathrm{~A}$ and C). 
Gao YH et al.

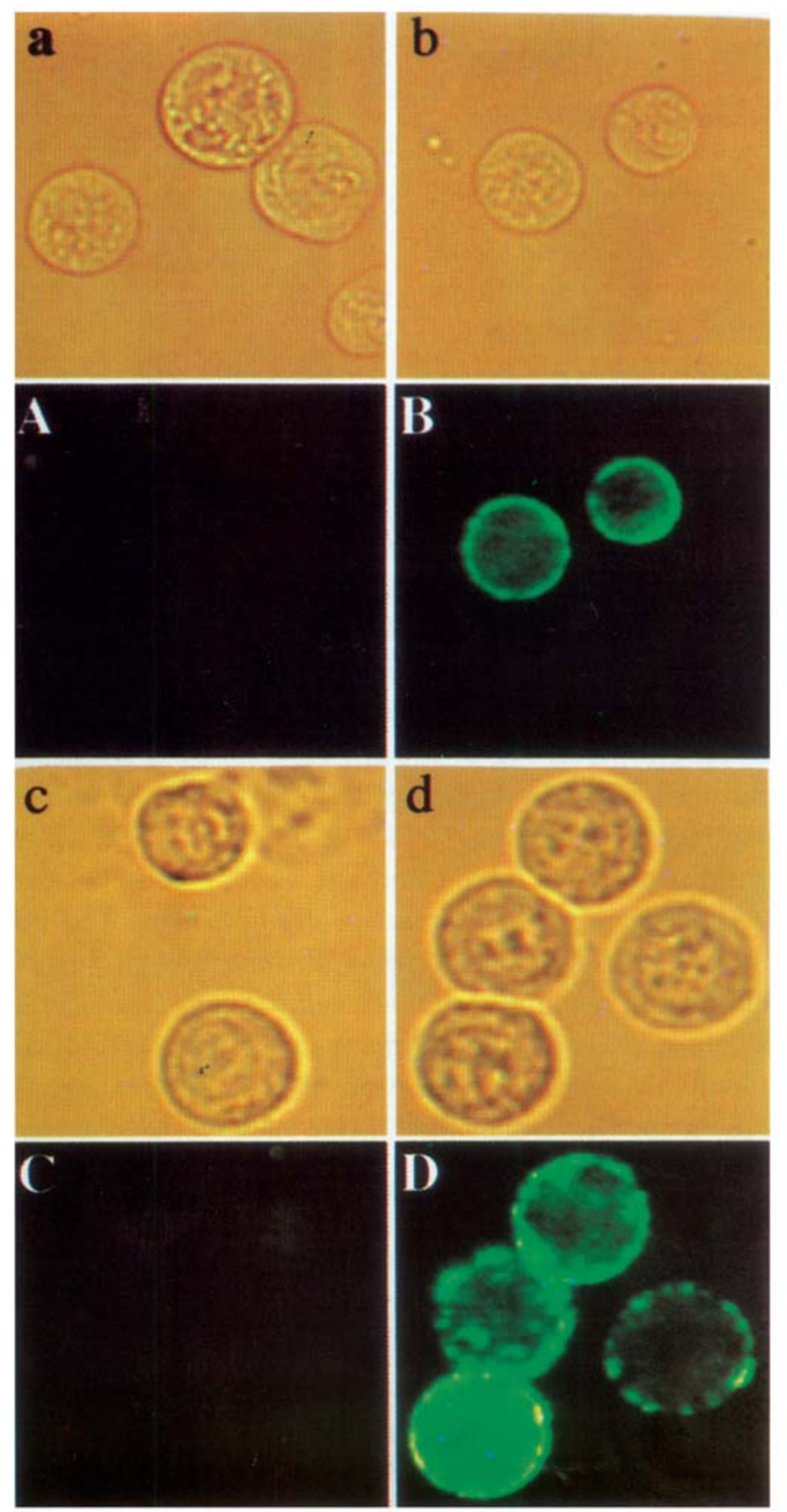


Expression of CTLA4 on Mm1 and its biological activity

Fig 4.

Images of binding sites of CTLA4Ig to B7-2 transfectant

A: confocal epifluorescence optical section;

B: conventional fluorescence micrograph.

B7-2 transfectant was subjected for observation.

Note that the staining was most prominent in the cell surface region observed by the epifluorescence optical section images with the confocal laser scanning microscope.
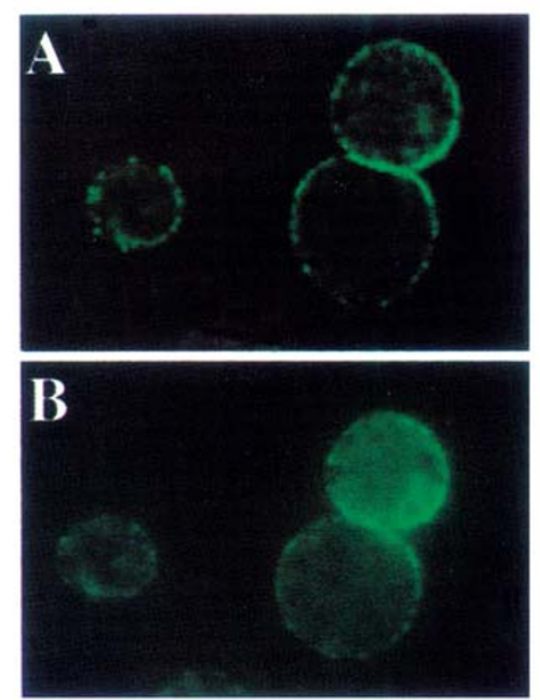

activation. Many studies have shown that the co-stimulatory signals through CD28 and/ or CTLA4 pathways are necessary for T-cell activation[1-3]. CTLA4Ig has been found to be able to block T-cell co-stimulation and allogeneic MLC when tested in vitro. BALB/c spleen cells were co-cultured with mitomycin C-treated C57BL/6 spleen cells in the medium containing CTLA4Ig from the transfected Mm1 cells. As shown in Fig 5, the spleen cells from BALB/c mice were activated when co-cultured with the spleen cells form C57BL/ 6 mouse and an isotope count of thymidine incorporated was $124836 \pm 5250 \mathrm{cpm}$. The spleen cell activation was completely inhibited by CTLA4Ig and the isotope count of thymidine incorporated was $83211 \pm 8604 \mathrm{cpm}$ in the presence of CTLA4Ig. The difference between CTLA4Ig-treated and untreated groups was absolutely significant $(\mathrm{p}<0$. 005). The control experiments using responder and stimulator cells from same BALB/c mouse were $95452 \pm 7280 \mathrm{cpm}$. Thus, CTLA4Ig from the transfected Mm1 cells, E522.7. 50 , seemed to be biologically active in inhibition of T-cell activation. The inhibition of MLC by CTLA4Ig from the transfected Mm1 cells resuted from the blockade of B7/CD28 co-stimulatory in T-cell.

\section{Conclusions}

To conclude, we have successfully introduced a murine CTLA4Ig gene into a macrophagic cell line $\mathrm{Mm} 1$ cells by the electroporation. CTLA4Ig from the transfected Mm1 cells could bind specifically to its ligands of B7 molecules and was biologically active. The expression of CTLA4Ig gene was stable and a high CTLA4Ig producing clone, E522.7.50, was obtained. The CTLA4Ig transfected Mm1 cells might be useful for the further biological and immunological studies in differentiation, maturation and functional activities of macrophages as well as investigation of CTLA4-related interactions of co-stimulation 
pathway in T-cell activation. Studies on therapeutic usage of the CTLA4Ig transfected $\mathrm{Mm} 1$ cells are ongoing and will be reported later.

\section{Fig 5.}

Inhibition of the MLC by CTLA4Ig from clone E522.7.50.

Spleen cells from BALB/c were incubated with mitomycin C-treated spleen cells from C57BL/6 mice in 1:2 dilution of the culture medium from E522.7.50, or in culture medium alone for $5 \mathrm{~d}$ and the T-cell proliferation was determined. Responder and stimulator cells from same BALB/c mouse were cocultured as the control group. Values represent the mean \pm S.D. of quadruplicates. Results shown were representive of three independent experiments.

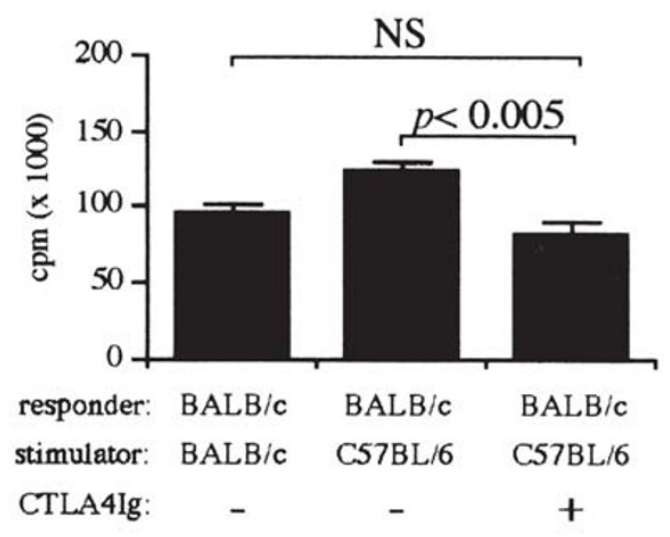

\section{ACKNOWLEDGMENT}

This research was funded by TARA (Tsukuba Advanced Research Alliance) special research grant of Tsukuba University (1996-1998), and was also supported in part by a grant from The Ministry of Education, Science, Sports and Culture (10650777).

\section{REFERENCES}

[1] Lane P. Regulation of T and B cell responses by modulating interactions between CD28/CTLA4 and their ligands, CD80 and CD86. Annals of the New York Academy of Sciences 1997; 815:392-400.

[2] Lenschow DJ, Walunas TL, Bluestone JA. CD28/B7 system of T cell costimulation. Annual Review of Immunology 1996; 14:233-58.

[3] Chambers CA, Allison JP. Costimulatory regulation of T cell function. Curr Opin Cell Biol 1999; 11: 203-10.

[4] Lu P, Wang YL, Linsley PS. Regulation of self-tolerance by CD80/CD86 interactions. Current Opinion in Immunology 1997; 9:858-62.

[5] Matsui T, Kurokawa M, Kobata T, et al. Autoantibodies to T cell costimulatory molecules in systemic autoimmune diseases. J Immunol 1999; 162:4328-35.

[6] Kemp EH, Ajjan RA, Husebye ES, et al. A cytotoxic T lymphocyte antigen-4 (CTLA-4) gene polymorphism is associated with autoimmune Addison's disease in English patients. Clin Endocrinol (Oxf) 1998; 49:609-13.

[7] Liu MF, Liu HS, Wang CR, Lei HY. Expression of CTLA-4 molecule in peripheral blood T lymphocytes from patients with systemic lupus erythematosus. J Clin Immunol 1998; 18:392-8.

[8] Azuma M, Ito D, Yagita H, et al. B70 antigen is a second ligand for CTLA-4 and CD28. Nature 1993; 366:76-9.

[9] Freeman GJ, Gribben JG, Boussiotis VA, et al. Cloning of B7-2: a CTLA-4 counter-receptor that costimulates human T cell proliferation [see comments]. Science 1993; 262:909-11.

[10] Freeman GJ, Borriello F, Hodes RJ, et al. Murine B7-2, an alternative CTLA4 counter-receptor that costimulates $\mathrm{T}$ cell proliferation and interleukin 2 production. Journal of Experimental Medicine 


\section{Expression of CTLA4 on Mm1 and its biological activity}

$1993 ; 178: 2185-92$.

[11] Lenschow DJ, Su GH, Zuckerman LA, et al. Expression and functional significance of an additional ligand for CTLA-4. Proceedings of the National Academy of Sciences of the United States of America 1993; 90:11054-8.

[12] Lane P, Gerhard W, Hubele S, Lanzavecchia A, McConnell F. Expression and functional properties of mouse B7/BB1 using a fusion protein between mouse CTLA4 and human gamma 1. Immunology 1993; 80:56-61.

[13] Gallon L, Chandraker A, Issazadeh S, et al. Differential effects of B7-1 blockade in the rat experimental autoimmune encephalomyelitis model. Journal of Immunology 1997; 159:4212-6.

[14] Daikh DI, Finck BK, Linsley PS, Hollenbaugh D, Wofsy D. Long-term inhibition of murine lupus by brief simultaneous blockade of the B7/CD28 and CD40/gp39 costimulation pathways. Journal of Immunology 1997; 159:3104-8.

[15] Chesnutt MS, Finck BK, Killeen N, Connolly MK, Goodman H, Wofsy D. Enhanced lymphoproliferation and diminished autoimmunity in CD4-deficient MRL/lpr mice. Clinical Immunology and Immunopathology 1998; 87:23-32.

[16] Finck BK, Linsley PS, Wofsy D. Treatment of murine lupus with CTLA4Ig. Science 1994; 265:12257.

[17] Schaub M, Issazadeh S, Stadlbauer TH, Peach R, Sayegh MH, Khoury SJ. Costimulatory signal blockade in murine relapsing experimental autoimmune encephalomyelitis. J Neuroimmunol 1999; 96:158-66.

[18] Cross AH, San M, Keeling RM, Karr RW. CTLA-4-Fc treatment of ongoing EAE improves recovery, but has no effect upon relapse rate. Implications for the mechanisms involved in disease perpetuation. J Neuroimmunol 1999; 96:144-7.

[19] Takiguchi M, Murakami M, Nakagawa I, et al. Blockade of CD28/CTLA4-B7 pathway prevented autoantibody-related diseases but not lung disease in MRL/lpr mice. Lab Invest 1999; 79:317-26.

[20] Kanematsu M, Suketa Y. Role of glucocorticoid on interleukin-6-induced cellular functions in the mouse macrophage cell line (Mm 1). Biochimica et Biophysica Acta 1997; 1357:192-200.

[21] Takagi K, Nukaya I, Yasukawa K, Suketa Y. Inhibitory mechanisms of antibody production by nitrogen oxides released from activated macrophages during the immune response: relationship to energy consumption. Immunology and Cell Biology 1994; 72:241-8.

[22] Takagi K, Hosaka T, Suketa Y. Effect of recombinant human interleukin-6 on nitrite production of mouse myeloid leukemia cells. Journal of Cellular Physiology 1991; 147:306-10.

[23] Michishita M, Yoshida Y, Uchino H, Nagata K. Induction of tumor necrosis factor-alpha and its receptors during differentiation in myeloid leukemic cells along the monocytic pathway. A possible regulatory mechanism for TNF-alpha production. Journal of Biological Chemistry 1990; 265:87519.

[24] Hirai K, Maeda M, Ichikawa Y. Development of annulate lamellae in mouse myeloblastic cell line when differentiated to macrophages. Journal of Electron Microscopy 1983; 32:13-9.

[25] Maeda M, Ichikawa Y. Spontaneous development of macrophage-like cells in a culture of myeloid leukemia cells. Gann 1973; 64:265-71.

[26] Yodoi J, Masuda T, Miyama M, Maeda M, Ichikawa Y. Interaction of lymphocytes and macrophage cell line cells (M1 cells). I. Functional maturation and appearance of Fc receptors im M1 cells. Cellular Immunology 1978; 39:5-17.

[27] Kyoizumi S, Noro N, Teshigawara K, Sakaguchi S, Masuda T. A cloned cell line, Mk1, possessing Ia antigens and accessory cell activity. Journal of Immunology 1982; 128:2586-94.

[28] Maeda M, Ichikawa Y, Azuma I. Differentiation and production of colony-stimulating factor induced by immunostimulants in a leukemia cell line. Journal of Cellular Physiology 1980; 105:33-8.

[29] Maeda M, Ichikawa Y. Production of a colony-stimulating factor following differentiation of leukemic myleoblasts to macrophages. Journal of Cellular Physiology 1980; 102:323-31. 
Gao YH et al.

[30] Stacey KJ, Ross IL, Hume DA. Electroporation and DNA-dependent cell death in murine macrophages. Immunology and Cell Biology 1993; 71:75-85.

[31] Kato K, Okumura K, Yagita H. Immunoregulation by B7 and IL-12 gene transfer. Leukemia 1997; 11: 572-6.

[32] Enomoto A, Kato K, Yagita H, Okumura K. Adoptive transfer of cytotoxic T lymphocytes induced by CD86-transfected tumor cells suppresses multi-organ metastases of C1300 neuroblastoma in mice. Cancer Immunology, Immunotherapy 1997; 44:204-10.

[33] Wang PC, Kimura S, Todokoro K, Okigaki T, Gao YH, Matsumura M. Stable Expression of Human Complement Receptor CR1 on Rat Renal Glomerular Epithelial Cell SGE1. Journa of Cellular Engineering 1996; 1:179-82.

[34] Laemmli UK. Cleavage of structural proteins during the assembly of the head of bacteriophage T4. Nature 1970; 227:680-5.

Received June-22-1999. ～Revised July-20-1999. ～Accepted July-23-1999. 